Research Article

\title{
Numerical Model on the Dynamic Behavior of a Prototype Kaplan Turbine Runner
}

\author{
Ming Zhang $(\mathbb{D}$ and Qing-Guang Chen \\ College of Mechanical and Electronic Engineering, Shandong University of Science and Technology, Qingdao, \\ Shandong 266590, China \\ Correspondence should be addressed to Qing-Guang Chen; chenqg@sdust.edu.cn
}

Received 11 August 2021; Accepted 2 September 2021; Published 8 September 2021

Academic Editor: Gengxin Sun

Copyright (c) 2021 Ming Zhang and Qing-Guang Chen. This is an open access article distributed under the Creative Commons Attribution License, which permits unrestricted use, distribution, and reproduction in any medium, provided the original work is properly cited.

\begin{abstract}
Experimental and numerical investigations of the modal behavior of a prototype Kaplan turbine runner in air have been conducted in this paper. The widely used roving accelerometer method was used in the experimental modal analysis. A systematic approach from a single blade model to the whole runner has been used in the simulation to get a thorough understanding. The experimental results show that all the detected modes concentrate their displacements on the impacted blade. The numerical results show that the modes of the single blade form different mode families of the runner, and each mode family corresponds to a narrow frequency band. Harmonic response analysis shows that, at the response peak point, the single blade excitation can only get mode shapes with concentrations on the exciting blade due to the superposition of the close modes in each mode family, which explains the experimental results well, while the mode superposition can be avoided by the order excitation method. With the reduction of the connection stiffness between the blades and hub/control system, the frequencies of most modes change from insensitive to more and more sensitive to the connection stiffness change, which results in a sensitive area and an insensitive area. Through comparison with the experimental results, it is indicated that the natural frequencies of the runner can probably be predicted by merging the runner into a whole body.
\end{abstract}

\section{Introduction}

Nowadays, hydropower contributes a lot to world electricity generation [1]. To meet the daily increasing demand for electricity, the power intensity in hydraulic turbines is promoted for both new construction and the updating of power plants, which makes the fluid pressures and velocities higher, resulting in higher hydraulic excitation forces. Moreover, among all types of renewable sources, hydropower is the only one with a wide range of power regulation with fast responses (20-100\% max. power, less than 1 minute) to offset the unstableness of the electricity grid caused by some other renewable sources, such as solar and wind energy. Therefore, hydraulic turbines operate at extreme off-design conditions and experience transient events much more times one day than before, which leads to even larger forces [2]. Such higher forces can produce higher vibration levels in the runners, which can cause fatigue damage and shorten their lifetime.

To avoid this situation and to prolong the lifetime of runners, thoroughly understanding the modal behavior of hydraulic turbine runners is very important. Kaplan turbines are one type of widely used hydraulic turbines, which can be seen mostly in low water head and large capacity hydropower plants [3], and the blades of Kaplan turbine runners can rotate to make the runner operate under high efficiencies for a wide range of operation. The rotation of the blades is controlled by a complex control system located inside the runner body. The typical structure of Kaplan turbines is shown in Figure 1. The excitation forces of Kaplan turbine runners can be both static and dynamic pressure loads $[4,5]$. The static pressure load is positively correlated with the flow rate passing through the runner, and the dynamic pressure load mostly comes from the rotor-stator interaction (RSI) $[6,7]$. 


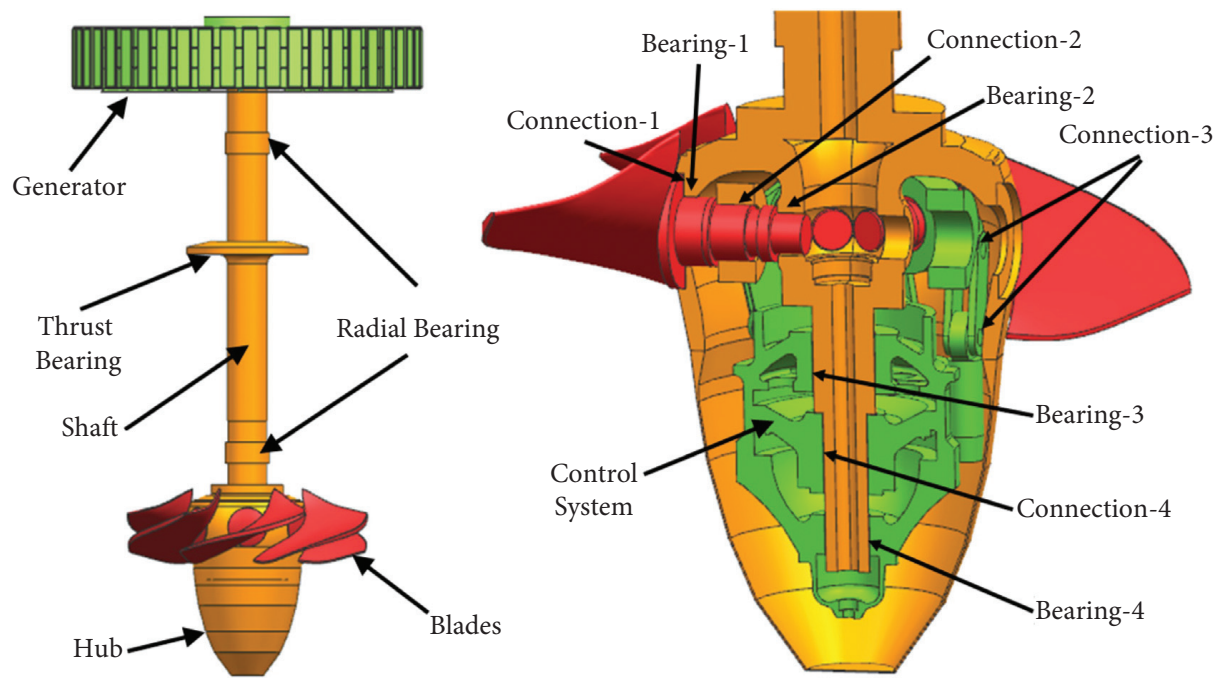

Figure 1: Structure of Kaplan turbines.

The modal behavior of Francis turbine runners and pump-turbine runners has been widely studied [8-14]. However, few investigations of the dynamic behavior of Kaplan turbine runners have been undertaken before. In another two papers $[15,16]$, the dynamic behavior of a single blade of a prototype Kaplan turbine was studied experimentally through the roving accelerometer method, but there is a lack of studies on the whole runner. There are some studies about the fluid-structure coupling vibration with only blade models of Kaplan turbine runners, but the influence of the hub/control system was not considered [17]. The complex connections between the blades and the runner body, as well as the complicated connections inside the control system, bring many difficulties to numerically study the modal behavior of Kaplan turbine runners. The typical connections in the Kaplan turbine are shown in Figure 1. These connections may have a large influence on the dynamic behavior of the runner because these connections can be loose, which may lower the support stiffnesses of the blades. Therefore, it is of great importance to know the connection stiffness levels, which can only be determined by experiments.

The structure of Kaplan turbines is similar to that of bladed disks [18-21], and they are all periodic and cyclic structures, though the disk part of Kaplan turbines (hub, control system, piston rod, etc.) can be more complicated than that of traditional blade-disk structures. For bladedisk structures, the modes of the single blade would form different mode families and each mode family consists of different ND (nodal diameter) modes. These different ND modes form a frequency band. For Kaplan turbines, the situations ought to be similar. However, because of the unknown connection stiffness levels, the width of the natural frequency band of each mode family is still unknown.

In this paper, the modal behavior of the same prototype Kaplan turbine in [15] has been studied experimentally and numerically after the reparation of the damaged blade. Unlike the study on a single blade [15], this research was conducted on the whole runner with one accelerometer on each blade to measure their responses. In the experiment, the roving accelerometer method was used and the peak-hold method was used to capture the mode shapes and natural frequencies. The influence of the connection stiffnesses on the modal behavior of the whole runner has been investigated numerically, and through comparison with the experimental results, the connection stiffness level is talked about. This paper is organized as follows: first, the theoretical background, experimental procedures, and numerical settings are introduced, then experimental and numerical results are presented, and finally, experimental and numerical results are compared and discussed.

\section{Theoretical Background}

2.1. Forced Response of a Structure. The governing equation of one structure in vibration can be expressed as

$$
[M] \ddot{x}(t)+[C] \dot{x}(t)+[K] x(t)=F(t),
$$

where $h(t), \dot{h}(t)$, and $\ddot{h}(t)$ are the displacement, velocity, and acceleration of each discrete point in the time domain, respectively; $[M],[C]$, and $[K]$ are, respectively, the mass, damping, and stiffness matrices; and $F(t)$ is the force vector applied on each discrete point in the time domain.

The frequency response function (FRF) can be obtained by applying Fourier transform to equation (1) in the frequency domain $(j \omega)$ :

$$
\{X(j \omega)\}=[H(j \omega)] \cdot\{F(j \omega)\},
$$

where $\{X(j \omega)\}$ and $\{F(j \omega)\}$ are the displacement and force vectors in the frequency domain, respectively, and $[H(j \omega)]$ is the conduction matrix between $\{X(j \omega)\}$ and $\{F(j \omega)\}$ and can be expressed as

$$
[H(j \omega)]=\sum_{r=1}^{n} \frac{j 2 \omega_{r} Q_{r}\{\varphi\}_{r}\{\varphi\}_{r}^{t}}{\left(\xi_{r}^{2}+\omega_{r}^{2}-\omega^{2}\right)-2 \xi_{r} j \omega} .
$$


$h_{a, b}$, any element of this matrix, can be seen as the response of discrete $a$ when a force is applied on another discrete point $b$ :

$$
h_{a, b}=\sum_{r=1}^{n} \frac{j 2 \omega_{r} Q_{r} \varphi_{a, r} \varphi_{b, r}}{\left(\xi_{r}^{2}+\omega_{r}^{2}-\omega^{2}\right)-2 \xi_{r} j \omega},
$$

where $\varphi_{a, r}$ and $\varphi_{b, r}$ are the modal displacements at the discrete point $a$ and $b$ for the $r$ mode.

2.2. Free Vibration of the Monocoupled Cyclic System. For a system with $N$ substructures, each substructure is seen as a lumped mass, for simplicity. The mass and stiffness of each substructure are $M$ and $K$, respectively, and each substructure is connected to the neighboring substructures with massless springs, whose stiffness is $k$. Only one freedom of the lumped mass is considered. The modal shapes of this system can be divided into two categories [21, 22]:

$$
\begin{aligned}
& U_{r}^{c}=\left\{1, \cos \alpha_{r}, \ldots, \cos (N-1) \alpha_{r}\right\}, \quad r=1, \ldots, \frac{N}{2}+1, \\
& U_{r}^{s}=\left\{0, \sin \alpha_{r}, \ldots, \sin (N-1) \alpha_{r}\right\}, \quad r=2, \ldots, \frac{N}{2},
\end{aligned}
$$

where $\alpha_{r}$ is the phase difference between every two neighboring substructures and can be expressed as $2 \pi(r-1) / N$. The eigenvalues of $r$ mode are

$$
\omega_{0 r}^{2}=\left[1+2 R^{2}\left(1-\cos \alpha_{r}\right)\right] \cdot \omega_{b}^{2},
$$

where $R^{2}=k / K$ is usually called the coupling effect and $\omega_{b}^{2}=$ $K / M$ is the circular natural frequency of each substructure. The lower and upper boundaries of the frequency band are

$$
\omega_{L}=\omega_{b} \omega_{U}=\sqrt{1+4 R^{2}} \omega_{b} .
$$

For $r$ equals 1 or $N / 2+1$, eigenvalues are singlet and all the rest are doublet. The ND (nodal diameter) of the mode $r$ is $r-1$.

\section{Experiment Setup}

Experimental modal analysis (EMA) [23] was undertaken on the same prototype Kaplan turbine runner in [15] after the reparation of the damaged blade. The head of the turbine is $34 \mathrm{~m}$, and the maximum power is $73 \mathrm{~mW}$. The machine is vertical and is restricted by a thrust bearing at the upper part of the shaft, one radial bearing on the turbine side and another radial bearing on the generator side. The runner has 6 blades with an outside diameter of $6 \mathrm{~m}$, and the nominal rotating speed is $125 \mathrm{rpm}$. One accelerometer was set on each blade, and 6 accelerometers were used in total. A single axis was used for each accelerometer. The accelerometers were attached perpendicularly to the blade profile to measure the response normal to the blade profile of each attached position. In the experiment, the roving accelerometer method was used. One blade was chosen for impact with a piezoelectric hammer (Dytran $5802 \mathrm{~A}, 220 \mu \mathrm{V} / \mathrm{N}$ ) normally to the blade profile of the impact position. The response was measured with the accelerometers (Kistler $8752 \mathrm{~A}, 100 \mathrm{mV} / \mathrm{g}$ ). Signals of the accelerometer and hammer in the time domain were transferred to a Bruel \& Kjaer (LAN XI Type 3053) acquisition system.

Totally 21 impact positions on the impacted blade were chosen for measurements, and the impact position was kept the same (near the leading edge). In this way, the mode shape of the blade for every natural frequency can be obtained. Through the responses at the same position on different blades, particularly the phase differences, the nodal diameter of one mode can be identified. Three impacts were undertaken in every measurement position, and the average value of them was used to calculate the frequency response function. The measurement positions on the impacted blade are shown in Figure 2. During the whole progress, the accelerometers on other nonimpacted blades were kept at the same position without movement.

The frequency response function (FRF), the relationship between the response of accelerometers and the force of the hammer, was computed by transforming equation (4) through the Fourier transformation. $8 \mathrm{~s}$ of response signal was taken for calculation, which determined the frequency resolution to be $0.125 \mathrm{~Hz}$. The coherence function was also computed between the accelerometer and the hammer to ensure the accuracy of the results. A value of the coherence function close to 1 means the noise and nonlinear effects are low. In this way, for each measurement point, one FRF was obtained. The peak hold method was used to extract natural frequencies and responses from each FRF. With all the responses of different measurement points, an operating deflection shape (ODS) [24] of the runner can be obtained, which can be used to analyze the mode shapes.

\section{Simulation Setup}

Simulations were conducted via Ansys Workbench 16.2 [25]. The FEM model includes the blades, hub, control system, hollow shaft, and generator. The numerical geometry profile was built from the sketch of the turbine. Therefore, there may be some geometry errors for some parts, particularly the blade profile. In [15], the numerical blade model was validated by the experiment. The structure material is stainless steel with a Young's modulus of $1.93 E+11 \mathrm{~Pa}$, density of $7750 \mathrm{~kg} / \mathrm{m}^{3}$, and poison ratio of 0.31 . The total weight of the generator was set to be $20300 \mathrm{~kg}$ (real value) through changing its material density. The restriction of radial bearings was simulated as elastic supports to the peripheral surface, and the foundation stiffness was set to be $6.524 \mathrm{~N} / \mathrm{mm}^{3}$ with an equivalent spring stiffness of $10^{10} \mathrm{~N} / \mathrm{m}$ around bearings, which is also used in some other similar studies [11]. When the machine is not in operation, the whole turbine is supported by the thrust bearing, and zero-displacement support was given to the bottom surface of the thrust bearing in the shaft axial direction. Bladedominated modes are usually not sensitive to the bearing stiffness due to the low deformation in the shaft, which can be seen in the following sections.

The connections in Figure 1 have been given some simplifications. The bearing-1 was shorted to the inner 

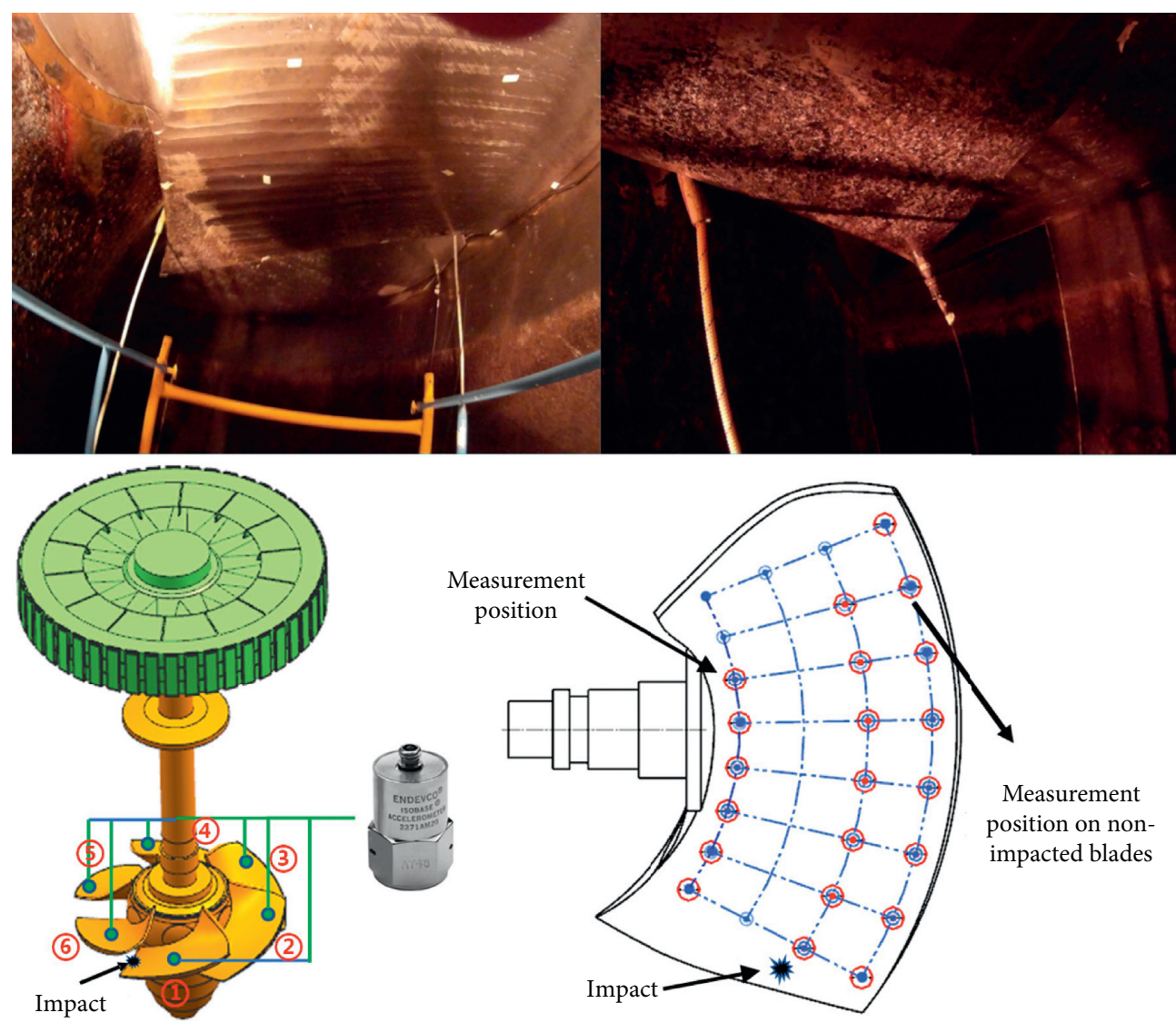

FIgURE 2: Experimental modal analysis.

surface of the hub, bearing- 2 was neglected and replaced with a small clearance, and a thin rod inside the shaft that is used to control the position of the control system has been omitted. Because the connections are too complicated, a thin layer was created at the location of connection-1 and bearing-1 in Figure 1 for each blade, and the support stiffness of the blade can be changed via the variation of Young's modulus of the layer material, and all other parts are merged as a whole body. In other words, the change of the layer stiffness acts as an equivalent support stiffness change due to all the connections. The mesh sensitivity has been strictly checked, and when the whole runner is simulated, about 67500 tetrahedral elements were used (Figure 3). A layer stiffness ratio is obtained by normalizing its Young's modulus to that of the stainless steel.

\section{Results and Discussions}

5.1. Experimental Results. Figure 4 shows the frequency response functions of different measurement points, including the points on the impacted blade and nonimpacted blades. Five modes are detected below $200 \mathrm{~Hz}$. The coherences of some measurement points are shown in Figure 5. At all the detected natural frequencies, the coherences are all close to 1 for both the measurement points on the impacted blade and nonimpacted blades. Generally, the mode shapes of a single blade can usually be bending (BD), torsion (TS), and in-plane (IP) modes. For torsion modes, they can usually be marked according to the number of its horizontal (perpendicular to the rod axis) nodal lines $\left(m_{H}\right)$ and vertical (parallel to the rod axis) nodal lines $\left(n_{V}\right)$. A nodal line is a line where the structure stands still on it. A mode $\left(m_{H}, n_{V}\right)$ means this mode has $m_{H}$ horizontal nodal lines (NLs) and $n_{V}$ vertical NLs. The mode names have been marked in Figure 4. In Bruel \& Kjaer, a numerical model consisting of 6 blades and one part of the turbine bearing has been built to present the mode shapes, which have been shown in Figure 6. The mode shapes on the impacted blade are presented especially.

In Figures 4 and 5, the measurement points on nonimpacted blades show low responses compared with those on the impacted blade at the same position for all the modes. The displacements of the measurement points on all the blades with the same position are plotted in Figure 7 (normalized to the maximum value of them for each mode). The blade numbers have been marked in Figure 2. As can be seen, the impacted blade (B1) has the highest displacement for all the modes. In other words, the ODSs of each mode all concentrate their displacements on the impacted blade.

\subsection{Numerical Results}

5.2.1. Influence of Support Stiffness on the Natural Frequencies of a Single Blade. To get a systematic understanding of the modal behavior of the runner, the influence of support stiffness on the natural frequencies of a single blade has been 


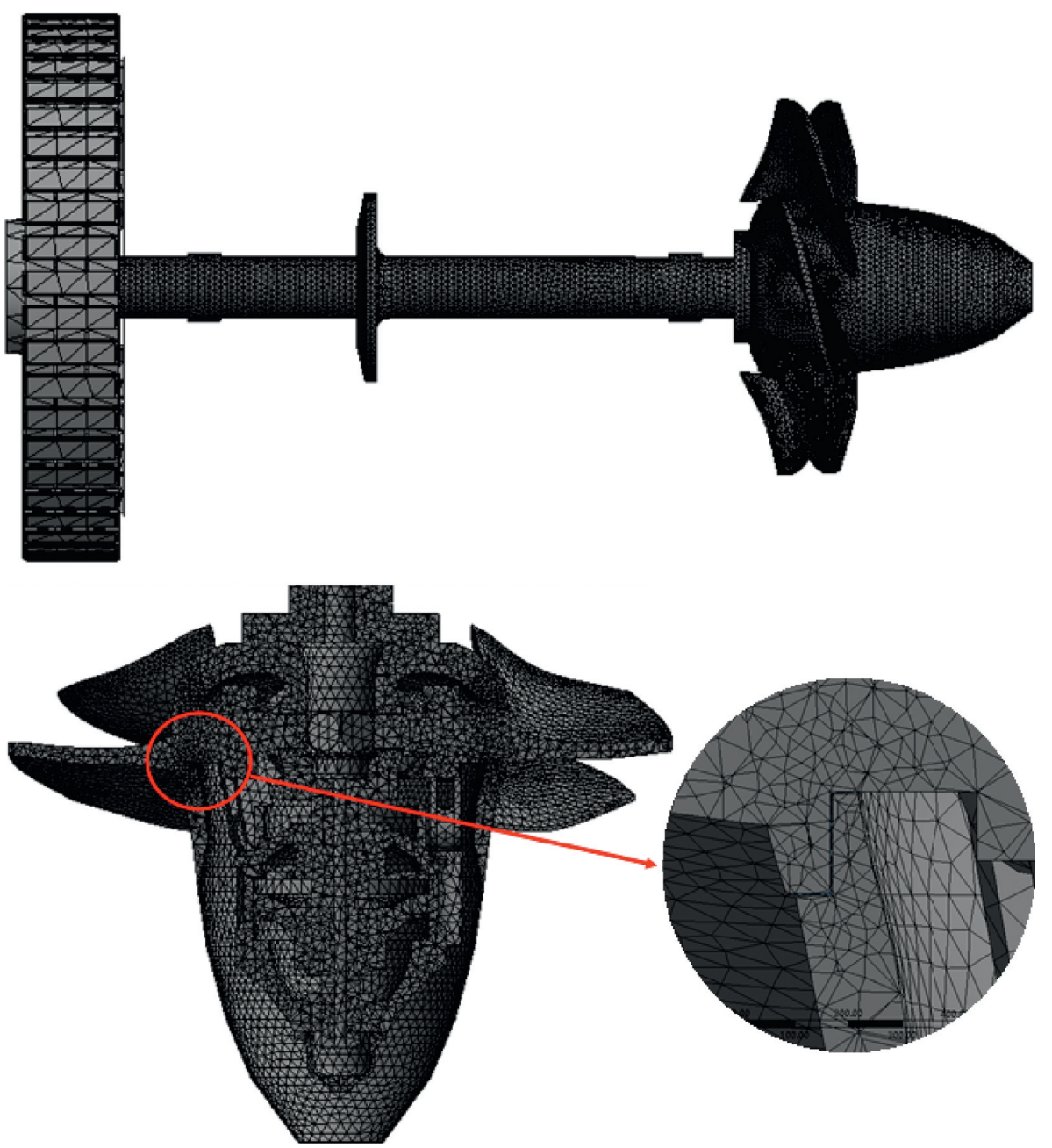

Figure 3: View of the mesh.

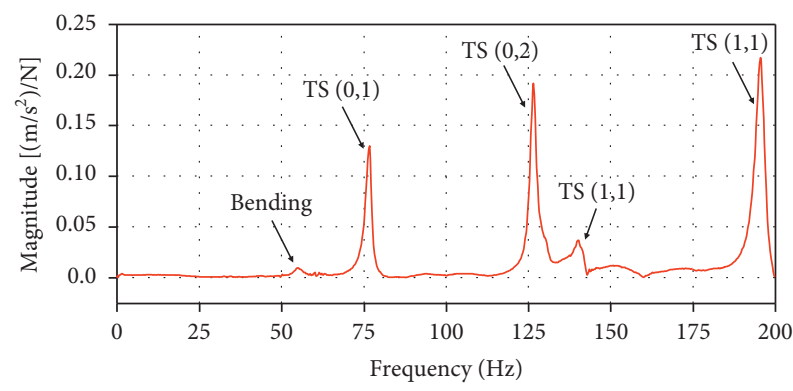

Impacted Blade

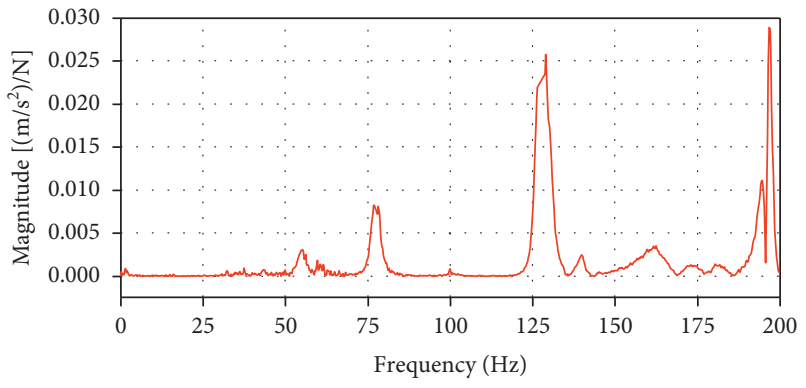

_ Non-Impacted Blade

FIGURE 4: Frequency response functions of a measurement point on the impacted blade and a measurement point on a nonimpacted blade. 


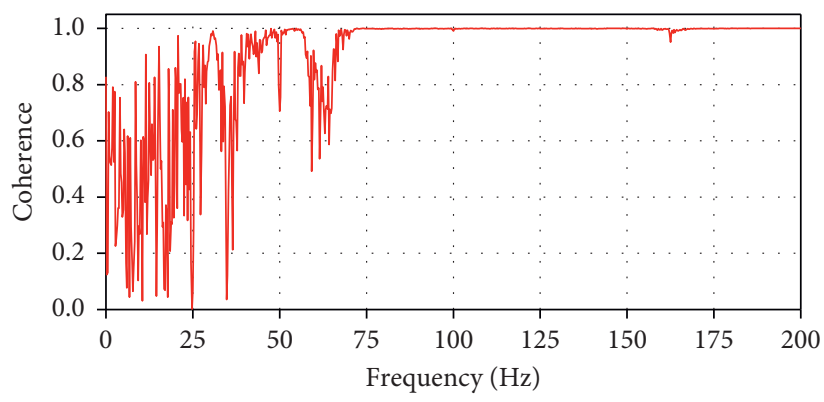

_ Impacted Blade

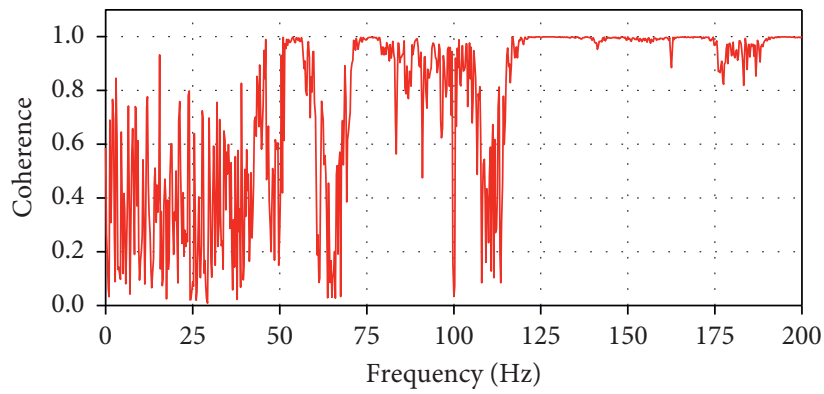

- Non-impacted Blade

Figure 5: Coherences between the hammer and different measurement points.

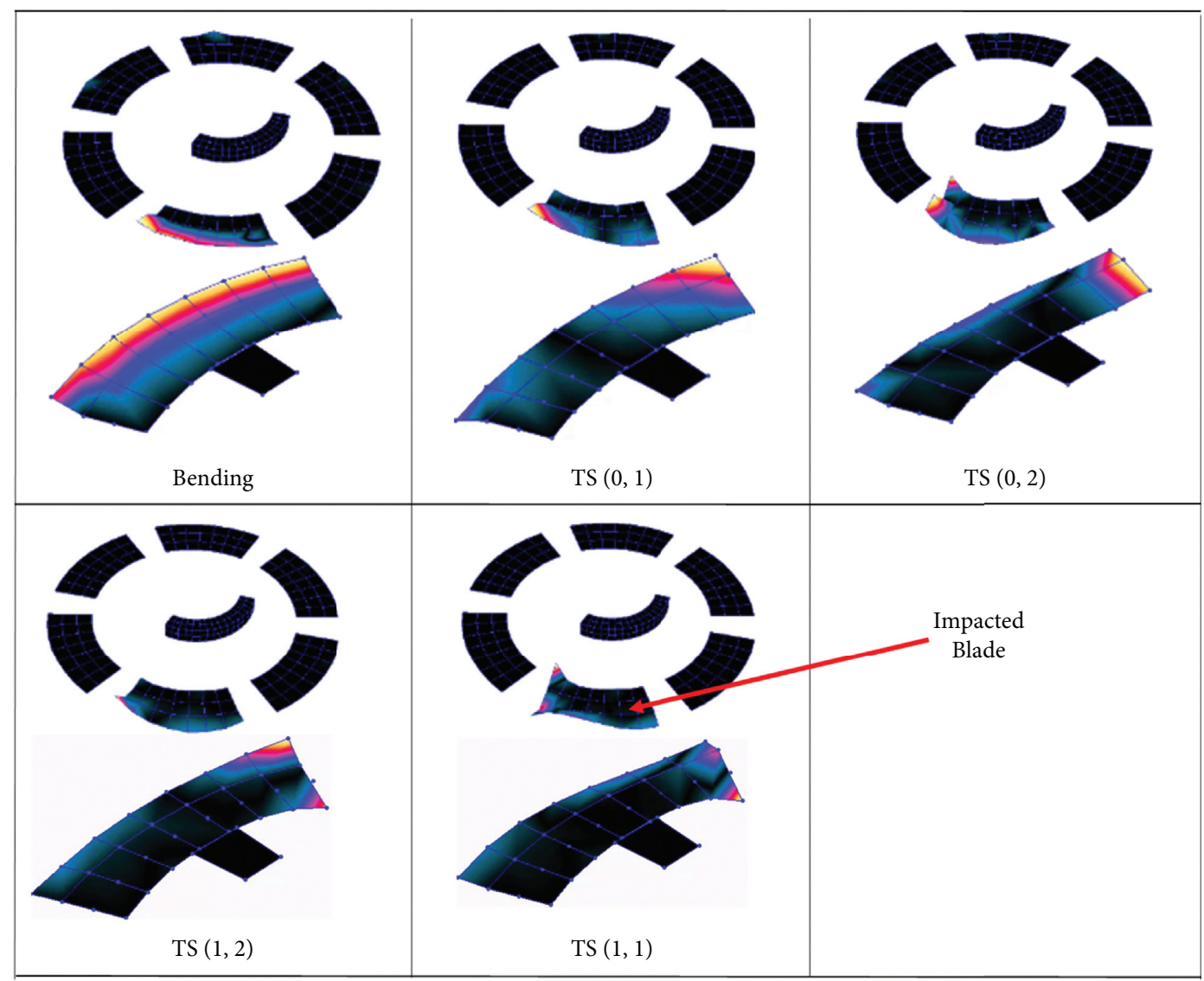

Figure 6: Operating deflection shapes (ODS) obtained from the experiment. (a) Bending, (b) $T(0,1),(\mathrm{c}) T(0,2),(\mathrm{d}) T(1,2)$, and (e) $T(1,1)$. 


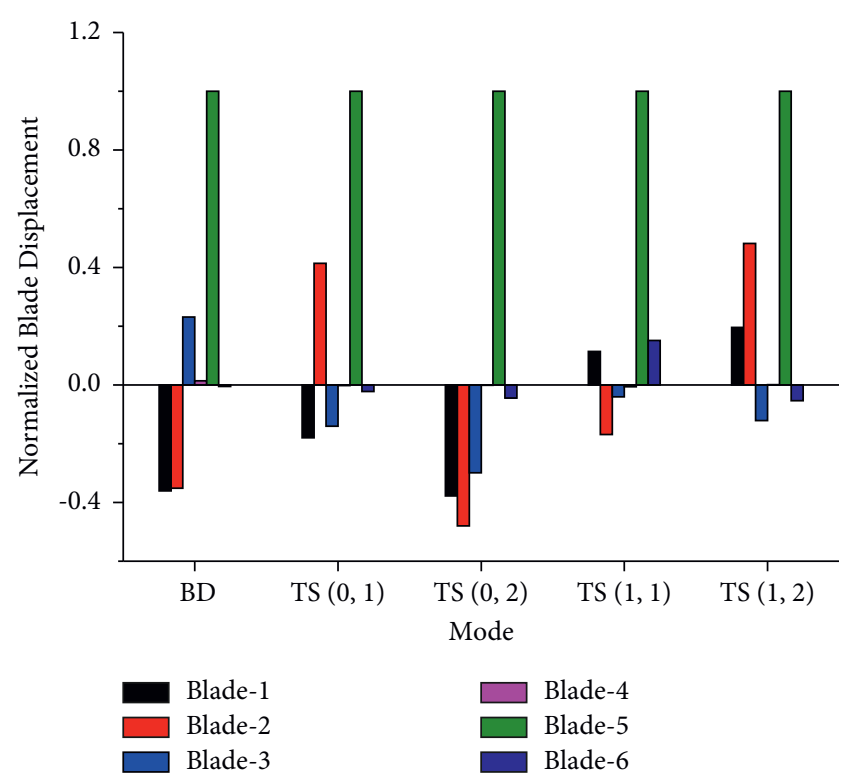

FIgURE 7: Normalized blade displacement of different modes.

first studied numerically. The investigated model (see Figure 8) is formed by a single blade and the layer. Fixed support is given to the rod end and the outside peripheral surface of the aforementioned layer. The fixed support at the end of the rod is similar to providing base support stiffness to the blade. The total support stiffness can be modified by changing the material stiffness of the layer. The first six modes of the blade under a layer stiffness ratio of 1.0 are shown in Figure 9. The bending mode and torsion modes have all been detected by the experiment, while the IP mode is not detected in the experiment probably because this mode mainly vibrates parallelly to the blade profile plane, but the accelerometers only measure the response in the direction perpendicular to the blade profile.

The frequencies of different modes under different layer stiffness ratios normalized (normalized to Young's modulus of stainless steel) to those under layer stiffness ratio 10.0 are shown in Figure 10. When the support stiffness is high enough, the frequencies of all the modes approach convergent values. The natural frequencies of all modes are not sensitive to the layer stiffness change at the beginning, but they may become more and more sensitive with the decrease of the layer stiffness.

5.2.2. Modal Behavior of the Whole Runner. According to equations (5) and (6), for this type of periodic and cyclic structures, the modes of the single blade form different mode families, and each mode family contains six modes, namely, the singlet $0 \mathrm{ND}$, doublet $1 \mathrm{ND}$ and $2 \mathrm{ND}$, as well as singlet $3 \mathrm{ND}$. Each mode family corresponds to a narrow frequency band. The numerical frequency bands of different mode families when the layer stiffness is 1.0, as well as the experimental frequency values of each mode, are shown in Table 1 . One mode of each mode family and the 6 modes of TS $(1,2)$ mode family are shown in Figure 11.
Generally, unlike that shown in equation (7), the appear sequences of different ND in each mode family are much more random because the interaction intensities between the blades and hub/control system of different ND modes are different. A higher interaction intensity activates more mass to vibrate together with the blades, thus lowering the natural frequency. These interaction differences are mainly determined by the movement motions of the blades of different ND modes. The interaction of the 1ND mode of the bending family is the strongest, which makes its frequency lowest in that mode family. From Figure 9, we can see that the deformation at the rod part of all modes is low, which determines the interactions for all mode families are not high generally, thus resulting in low interaction differences of different ND modes and narrow frequency bands for all the mode families. Among these mode families, the interactions of the bending mode family usually are the strongest, which makes the width of its frequency band the highest.

The influence of layer stiffness ratio on the natural frequencies of all ND modes in the bending and TS $(0,2)$ mode family is shown in Figure 12 (normalized to those under layer stiffness ratio 1.0). A lower layer stiffness usually means a lower frequency for most modes, which is the same as that of the single blade model. However, for some modes, e.g., $0 \mathrm{ND}$ of the TS $(1,2)$ mode family, the changes may not be monotonous. This is possible because though the decrease of the layer stiffness lowers the support stiffnesses of the blades, it also lowers the coupling stiffness in equation (7), which activates less mass to vibrate together with the blades, thus increasing the frequencies, particularly when the frequencies of the blades are not sensitive to the layer stiffness change. The same is observed with those of the single blade, when the layer stiffness is lower than some values, as marked in Figure 12, the frequencies of all modes become very sensitive to the layer stiffness change. Generally, according to the sensitivity to the support stiffness change, the frequency changes can usually be classified into the sensitive area and insensitive area, which have been marked in Figure 12.

5.2.3. Harmonic Response Analysis of the Runner. In the experiment, the displacements of all the detected modes concentrate on the impacted blade and no mode shows a pure harmonic waveform of any ND. At the same time, there is usually only one mode that is detected for each mode family through the peak-hold method. From Table 1, the natural frequencies of all ND modes in each mode family are very close. In the experiment, the roving accelerometer method was used, which is similar to exit the runner on a single blade. According to equation (4), because of the close natural frequencies and the effect of damping, it is very difficult for the response to be dominated by only one mode. In this section, this point will be tested through the harmonic response analysis. First, a $1 \mathrm{~N}$ force excitation on blade 1 at the tipping point of the trailing edge in the shaft axial direction was implemented. The forced response and the operating deflection shape (ODS) at the frequency with peak response are shown in Figure 13(a). 

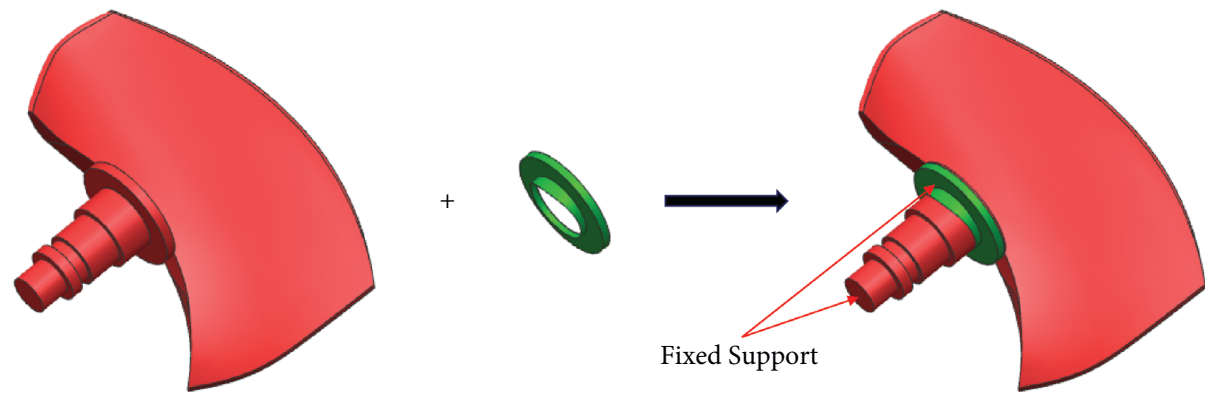

FIGURE 8: Single blade model.

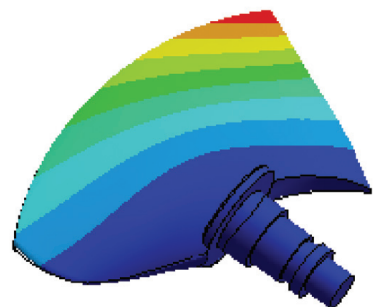

Bending

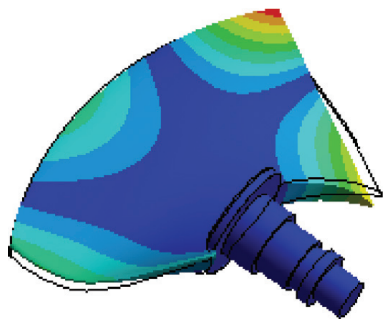

$\mathrm{T}(1,1)$

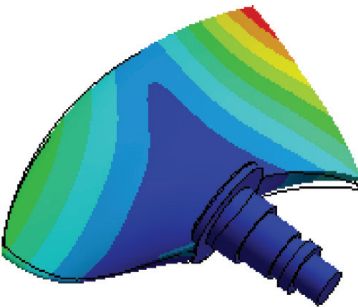

$\mathrm{T}(0,1)$

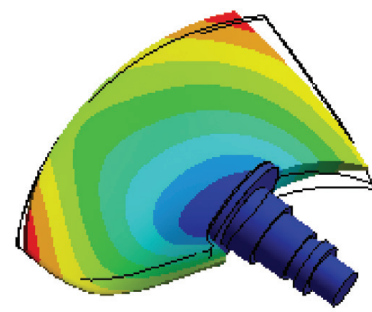

IP

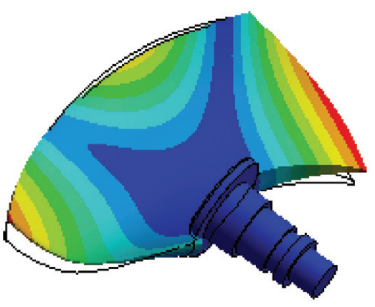

$\mathrm{T}(0,2)$

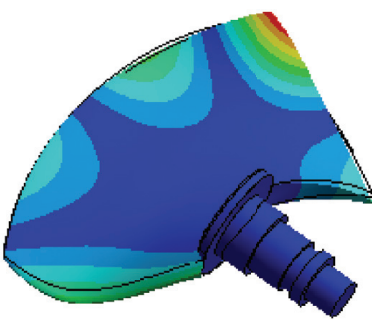

$\mathrm{T}(1,2)$

Figure 9: Mode shapes of the single blade model. (a) Bending, (b) $T(0,1),(c) T(0,2),(d) T(1,1),(e)$ IP, and (f) $T$ (1, 2).

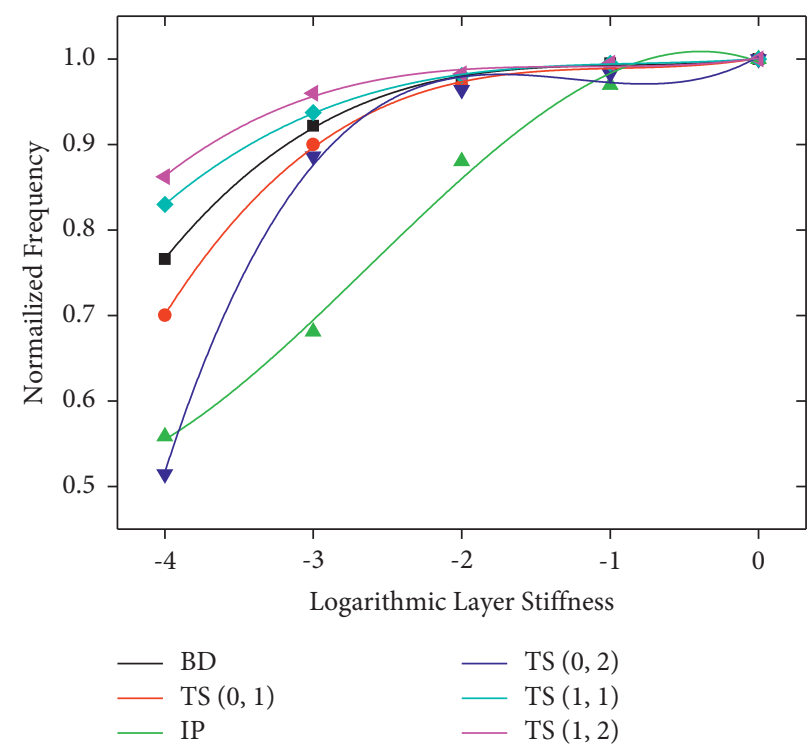

Figure 10: Influence of layer stiffness on the natural frequencies. 
Table 1: Experimental and numerical frequencies.

\begin{tabular}{lcr}
\hline Mode & Simulation $(\mathrm{Hz})$ & Experiment $(\mathrm{Hz})$ \\
\hline BD & $51.84-60.94$ & 55.42 \\
TS $(0,1)$ & $73.43-77.84$ & 77.39 \\
IP & $91.78-97.81$ & Not detected \\
TS $(0,2)$ & $114.29-123.75$ & 126.25 \\
TS $(1,1)$ & $142.49-150.21$ & 141.31 \\
TS $(1,2)$ & $191.15-197.37$ & 195.39 \\
\hline
\end{tabular}

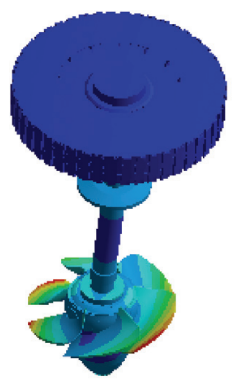

Bending

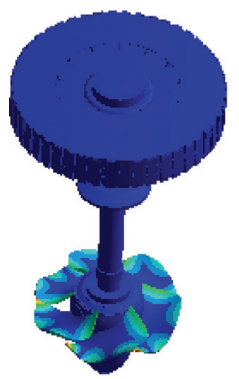

0ND

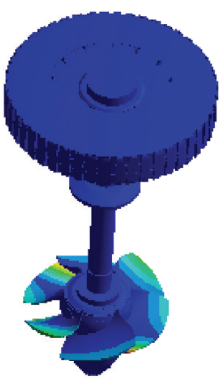

$\mathrm{T}(0,1)$

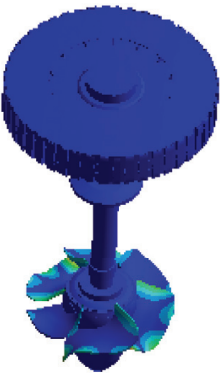

1ND-1

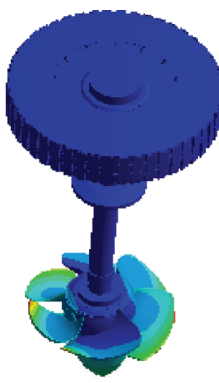

IP

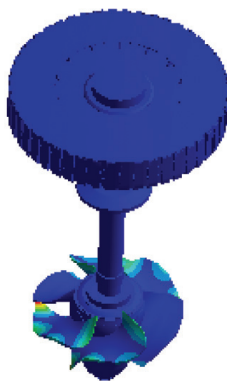

1ND-2

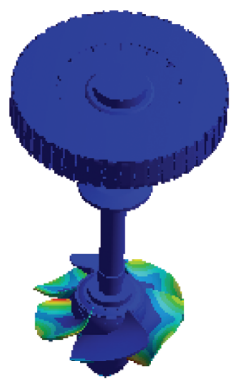

$\mathrm{T}(0,2)$

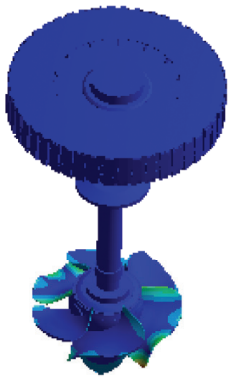

2ND-1

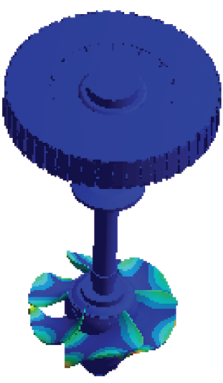

$\mathrm{T}(1,1)$

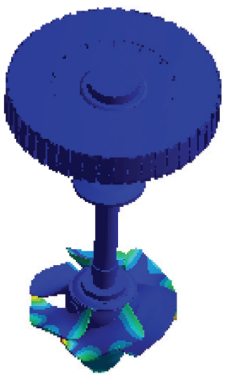

2ND-2

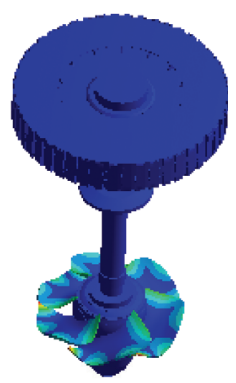

$\mathrm{T}(1,2)$

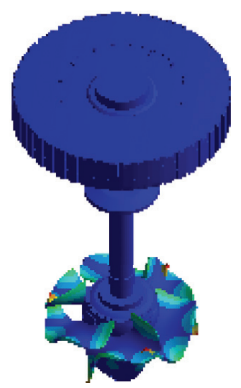

$3 \mathrm{ND}$

FIGURE 11: Different mode families (a) and the modes of the TS $(1,2)$ mode family (b).
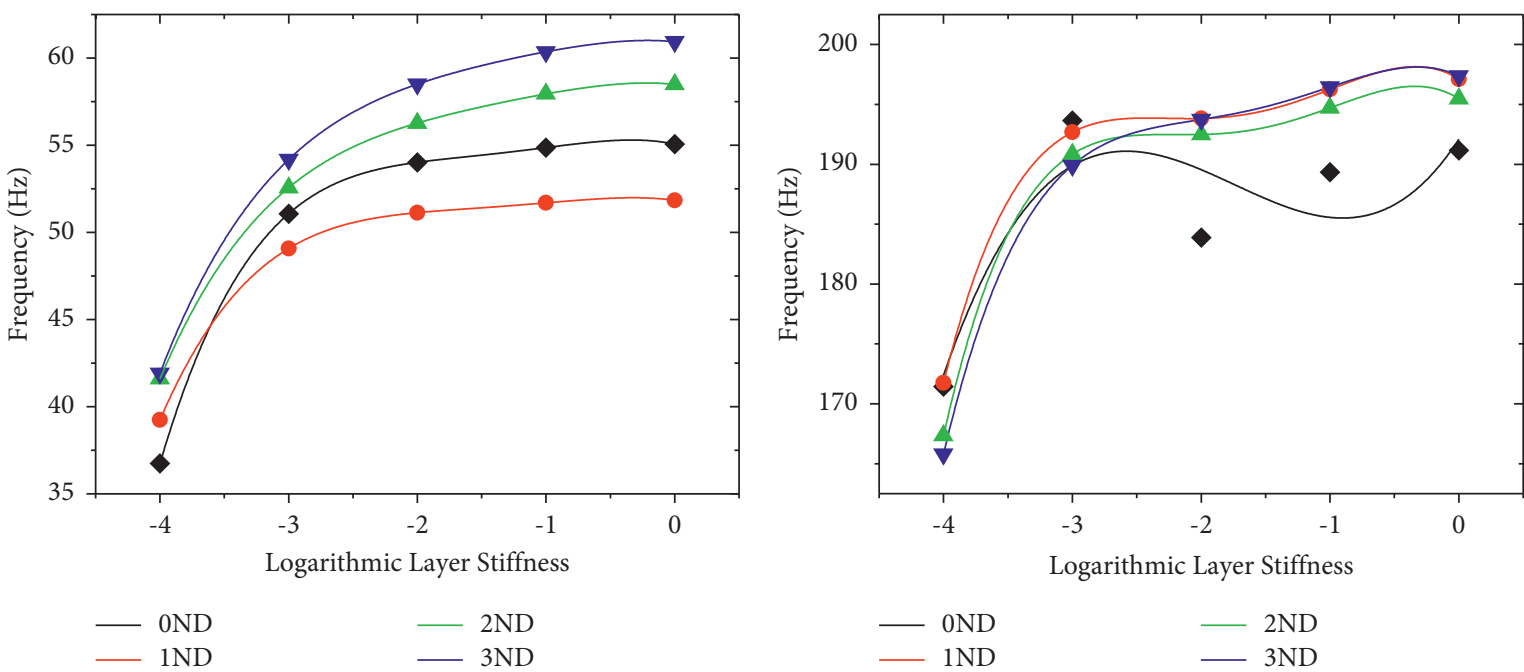

(a)

(b)

FIGURE 12: Influence of layer stiffness on the normalized frequency of all ND modes in the bending $(a)$ and TS $(1,2)(b)$ mode family. 

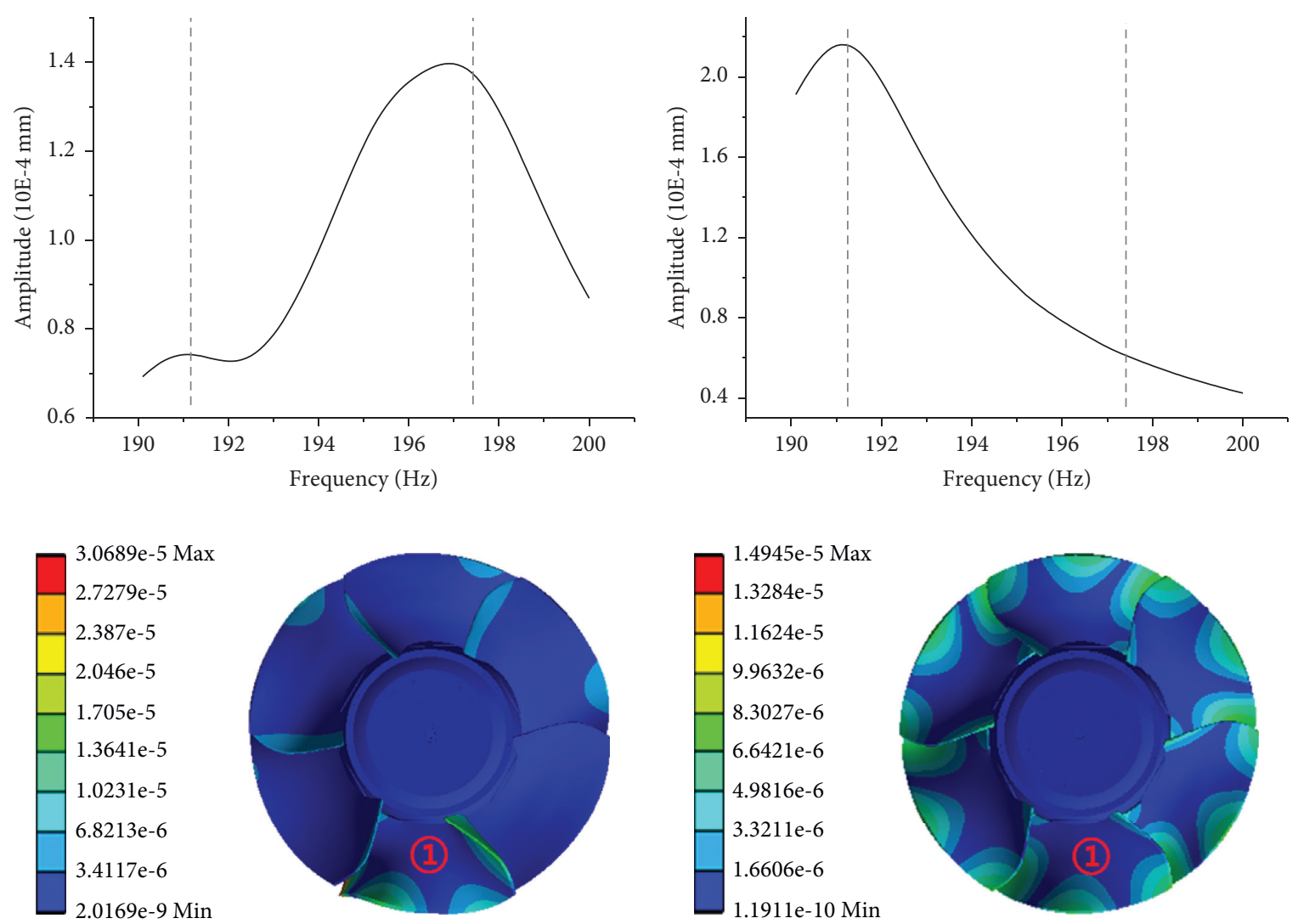

(a)

(b)

FIGURE 13: Forced response of the TS $(1,2)$ mode family under single blade excitation (a) and order excitation (b).

The upper and lower limits of the natural frequency band of TS $(1,2)$ are also marked in Figure 13 using grey dashed lines. When exciting a single blade, the ODS of the peak response indeed concentrates at the impacted blade 1 with some low displacements on blade-4, which is the same with the experimental results, and this response appears at a frequency between the upper and lower frequency limits. Though the peak response is not dominated by a pure mode, from equation (4), the response certainly has relationships with the modal displacement of the impact point, and a higher modal displacement is prone to produce a higher response. Under this situation, the ODS of the peak response cannot have low responses on the impacted blade, and obviously, a shape with a concentration on the impacted blade is more prone to produce high responses. Of course, the final ODS of the peak response is still restricted by equation (4). Because of the severe mode superposition, there is usually only one peak for each mode family, as shown in Figures 4 and 13. Under this situation, even some multi-DOF mode extraction methods, such as the complex exponential (CE) method $[18,19]$, may still be incapable to get the natural frequencies and modes shapes in each mode family.

Here, we recommend the order excitation method to study the modal behavior of Kaplan turbines. For turbine structures, under an order excitation with a certain ND, one mode can only be excited by the corresponding ND excitation. When the modes of a turbine structure are close, this method together with the peak-hold method can be used to avoid mode superposition to get the mode shapes and their frequencies, which can be seen in $[12,19,20]$. 0ND order excitation with a $1 \mathrm{~N}$ excitation force was applied on each blade. The TS $(1,2)$ mode family under the layer stiffness ratio 1.0 was still chosen for testing, and the experimental damping ratio was applied. The forced response and the operating deflection shape (ODS) at the frequency with peak response are shown in Figure 13(b). As expected, the 0 ND mode shape is excited at the peak response because other ND modes cannot be excited by this order excitation, which makes the exited mode dominant at the peak response. Therefore, the roving hammer/accelerometer method, which has been widely applied to investigate the modal behavior of other types of hydraulic turbine runners [8, 10], is not suitable to get the mode shapes and natural frequencies of blade-dominated modes of Kaplan turbines due to the close natural frequencies in each mode family, and they are better to be obtained through the order excitation method.

When using the roving accelerometer/hammer method in the experimental modal analysis, a harmonic response analysis may be needed in the simulation to match the frequency of the peak response of each mode family with that of experimental results to determine the equivalent connection stiffness level. When using the order excitation method, because of the nonmonotonous frequency changes of some ND modes with the decrease of the support stiffness, 
all ND modes in each mode family may need to be considered. If the connection stiffness is demonstrated to be located at the insensitive area, the runner merged as a whole body can be used to estimate the runner frequencies, and this can bring great conveniences. Though the numerical geometry errors bring some difficulties to determine the real connection stiffness levels, from the detailed comparisons of natural frequencies and modal shapes between the experimental and numerical results for the studied turbine blade in [16], the geometry errors ought to be limited. Together considering all the experimental frequencies in Table 1 fall into the numerical frequency bands or slightly higher than the upper limits of numerical frequency bands, it seems that the runner merged as a whole body can still be used to estimate the natural frequencies of the real runner.

\section{Conclusions}

The modal behavior of a prototype Kaplan turbine runner is investigated experimentally and numerically in this paper. The roving accelerometer method combined with the peakhold method has been used in the experimental modal analysis. A systematic approach from a single blade model to the whole runner has been used in the simulation. The experimental results show that the displacements of all detected modes concentrate on the impacted blade. The simulation shows that, for Kaplan turbines, the modes of single blade form different blade-dominated mode families. Each mode family contains 6 (the number of the blades) modes, including the singlet $0 \mathrm{ND}$, doublet $1 \mathrm{ND}$ and $2 \mathrm{ND}$, as well as singlet 3ND. Each mode family corresponds to a narrow frequency band. Because of the close natural frequencies in each mode family, the single blade excitation can only get a mode shape with a concentration on the exciting blade due to severe mode superposition. The roving accelerometer/hammer method is the same as single blade excitation, which makes the modes detected in the experiment concentrate their displacements on the impacted blade. However, the order excitation method can be used to get the natural frequencies, mode shapes, and damping ratios of different ND modes in each mode family with little influence of the mode superposition.

A systematic approach from the single blade model to the whole runner model was used in the simulation. The numerical results of the single blade model show that when the connection stiffness is higher than some values, the frequencies of all modes are not sensitive to the connection stiffness change and approach to convergent values. With the reduction of the connection stiffness, the frequencies of most modes become more and more sensitive to the connection stiffness change and may fall to sensitive areas when the support stiffness is lower than some values. This is also applicable to the whole runner model. If the connection level lies at the insensitive area, merging the whole runner into a whole body can be a quick method to estimate the natural frequencies of the prototype turbine runner, which can bring great conveniences. For the runner studied, after the comparison with experimental results, the runner merged as a whole body is demonstrated to be able to predict the natural frequencies of the real runner with limited errors.

In the future, the order excitation method can be used to investigate the natural frequencies, mode shapes, and damping ratios of different ND modes in each mode family. Though the study in this paper shows that the natural frequencies can be approximately predicted by merging the runner as a whole body for the investigated runner, it is better to be validated for more runners to get more solid conclusions.

\section{Abbreviations}

$x(t): \quad$ Vibration displacement at an instant time

DOF: Degree of freedom

FRF: $\quad$ Frequency response function

$[H(j \omega)]$ : Transfer matrices in the frequency domain

$F(t)$ : $\quad$ Force applied on each DOF in the time domain

$\xi_{r}: \quad$ Damping ratio

$Q_{r}: \quad$ The scale factor of mode $r$

$\{\varphi\}_{r}: \quad$ Mode shape of mode $r$

$\omega_{r}$ : The natural frequency of the corresponding mode

FFT: $\quad$ Fast Fourier transform

$k_{c}$ : $\quad$ Coupling stiffness

$U_{r}^{c}: \quad$ Cosine category of modal shapes

$U_{r}^{s}: \quad$ Sine category of modal shapes

$r: \quad$ Eigen order

ND: $\quad$ Nodal diameter

$R: \quad$ Coupling stiffness

$\omega_{L}: \quad$ Lower boundary of the passband

$\omega_{U}: \quad$ Upper boundary of the passband

$\alpha_{r}: \quad$ Phase difference between neighboring substructures

$\omega_{0 r}: \quad$ Natural frequency of the $(r-1)$ ND mode

$\omega_{b}$ : Natural frequency of the substructure

$m_{H}$ : Number of the horizontal nodal lines

$\lambda: \quad$ Frequency reduction ratio of the localized mode

$n_{V}: \quad$ Number of the vertical nodal lines.

\section{Data Availability}

No data were used to support this study.

\section{Conflicts of Interest}

The authors declare that they have no conflicts of interest.

\section{Acknowledgments}

The authors wish to acknowledge the economic support received from the Natural Science Foundation of Shandong Province (No. ZR2018MEE036) and the Project of Shandong Province Higher Educational Young Innovative Talent Introduction and Cultivation Team (performance enhancement of deep coal mining equipment). 


\section{References}

[1] IEA, Key Electricity Trends 2017 Based on Monthly Data. Monthly Electricity Statistics, IEA, Paris, France, 2018.

[2] C. Trivedi, B. Gandhi, and C. J. Michel, "Effect of transients on Francis turbine runner life: a review," Journal of Hydraulic Research, vol. 51, no. 2, pp. 121-132, 2013.

[3] H. R. Krishna, Hydraulic Design of Hydraulic Machinery, Avebury, Wiltshire, UK, 1997.

[4] S. Liu, S. Li, and Y. Wu, "Pressure fluctuation prediction of a model kaplan turbine by unsteady turbulent flow simulation," Journal of Fluids Engineering, vol. 131, no. 10, Article ID 101102, 2009.

[5] L. Zhou, Z. Wang, R. Xiao, and Y. Luo, "Analysis of dynamic stresses in kaplan turbine blades," Engineering Computations, vol. 24, no. 8, pp. 753-762, 2007.

[6] C. G. Rodriguez, E. Egusquiza, and I. F. Santos, "Frequencies in the vibration induced by the rotor stator interaction in a centrifugal pump turbine," Journal of Fluids Engineering, vol. 129, no. 11, pp. 1428-1435, 2007.

[7] G. A. Gerolymos, G. J. Michon, and J. Neubauer, “Analysis and application of chorochronic periodicity in turbomachinery rotor/stator interaction computations," Journal of Propulsion and Power, vol. 18, no. 6, pp. 1139-1152, 2002.

[8] C. G. Rodriguez, E. Egusquiza, X. Escaler, Q. W. Liang, and F. Avellan, "Experimental investigation of added mass effects on a Francis turbine runner in still water," Journal of Fluids and Structures, vol. 22, no. 5, pp. 699-712, 2006.

[9] Q. W. Liang, C. G. Rodríguez, E. Egusquiza, X. Escaler, M. Farhat, and F. Avellan, "Numerical simulation of fluid added mass effect on a francis turbine runner," Computers \& Fluids, vol. 36, no. 6, pp. 1106-1118, 2007.

[10] E. Egusquiza, C. Valero, Q. Liang, M. Coussirat, and U. Seidel, "Fluid added mass effect in the modal response of a pumpturbine impeller," in Proceedings of the ASME 2009 International Design Engineering Technical Conferences and Computers and Information in Engineering Conference, American Society of Mechanical Engineers (ASME), San Diego, California, USA, August 2009.

[11] D. Valentín, D. Ramos, M. Bossio, A. Presas, E. Egusquiza, and C. Valero, "Influence of the boundary conditions on the natural frequencies of a Francis turbine," IOP Conference Series: Earth and Environmental Science, vol. 49, 2016.

[12] P. T. K. Østby, K. Sivertsen, J. T. Billdal, and B. Haugen, "Experimental investigation on the effect off near walls on the eigen frequency of a low specific speed francis runner," Mechanical Systems and Signal Processing, vol. 118, pp. 757766, 2019.

[13] A. Presas and Z. W. Wang, "Analysis of the dynamic response of pump-turbine runners-part I: experiment," IOP Conference Series: Earth and Environmental Science, vol. 15, no. 5, 2012.

[14] M. Zhang, D. Valentin, C. Valero, M. E. Montagut, and W. Zhao, "Numerical study on the dynamic behavior of a francis turbine runner model with a crack," Energies, vol. 11, no. 7, 2018.

[15] M. Zhang, D. Valentín, C. Valero, M. Egusquiza, and E. Egusquiza, "Failure investigation of a kaplan turbine blade," Engineering Failure Analysis, vol. 97, pp. 690-700, 2019.

[16] M. Zhang, D. Valentin, C. Valero, A. Presas, M. Egusquiza, and E. Egusquiza, "Experimental and numerical investigation on the influence of a large crack on the modal behaviour of a kaplan turbine blade," Engineering Failure Analysis, vol. 109, Article ID 104389, 2020.
[17] M. Zhang and Q.-G. Chen, "Influence of internal blade-interactions on the added mass and added damping of a prototype kaplan turbine runner," Alexandria Engineering Journal, 2021, In press.

[18] M. P. Castanier and C. Pierre, "Modeling and analysis of mistuned bladed disk vibration: current status and emerging directions," Journal of Propulsion and Power, vol. 22, no. 2, pp. 384-396, 2006.

[19] M. J. Kruse and C. Pierre, "An experimental investigation of vibration localization in bladed disks: part I-free response," in Proceedings of the ASME 1997 International Gas Turbine and Aeroengine Congress and Exhibition, American Society of Mechanical Engineers Digital Collection, Orlando, FL, USA, June 1997.

[20] M. J. Kruse and C. Pierre, "An experimental investigation of vibration localization in bladed disks: part II-forced response," in Proceedings of the ASME 1997 International Gas Turbine and Aeroengine Congress and Exhibition, American Society of Mechanical Engineers (ASME), Orlando, FL, USA, June 1997.

[21] X. Fang, J. T. Tang, E. H. Jordan, and K. D. Murphy, "Crack induced vibration localization in simplified bladed-disk structures," Journal of Sound and Vibration, vol. 291, no. 1-2, pp. 395-418, 2006.

[22] S.-T. Wei and C. Pierre, "Localization phenomena in mistuned assemblies with cyclic symmetry part I: free vibrations," Journal of Vibration and Acoustics, vol. 110, no. 4, pp. 429438, 1988.

[23] P. Avitabile, "Experimental modal analysis," Sound and Vibration, vol. 35, no. 1, pp. 20-31, 2001.

[24] B. J. Schwarz and M. H. Richardson, Introduction to Operating Deflection Shapes, Vibrant Technology Inc., Jamestown, NY, USA, 1999.

[25] P. C. Kohnke, Finite Element Systems, Ansys, Canonsburg, PA, USA, 1982. 